\title{
Magnetic Field Effects on In-Medium r Dissociation
}

\author{
Johannes Hoelck ${ }^{1, a}$, Felix Nendzig ${ }^{1}$, and Georg Wolschin ${ }^{1}$ \\ ${ }^{1}$ Institut für Theoretische Physik, Universität Heidelberg, Germany, EU
}

\begin{abstract}
The electromagnetic fields during relativistic heavy ion collisions are calculated using a simple model which characterises the emerging quark-gluon medium by its conductivity only. An estimate of the average magnetic field strength experienced by the $\mathrm{b} \overline{\mathrm{b}}$ mesons produced in the collision is made. In a sufficiently strong magnetic field, the individual spins of $b \bar{b}$ mesons can align with the field leading to quantum mixing of the singlet and triplet spin configurations. The extent of this intermixture, however, is found to be negligible at field strengths occurring in heavy ion collisions at LHC energies.
\end{abstract}

\section{Introduction}

The suppression of $\Upsilon$ meson production in heavy ion collisions can be used as a probe for the properties of the quark-gluon medium expected to be formed during these events. Being part of the more massive $b \bar{b}$ meson family, the $\Upsilon$ is less prone to recombination than the relatively lightweight $J / \psi$ meson which might offer the opportunity for a cleaner examination of the underlying suppression mechanisms.

We have interpreted recent LHC data [1] for the $\Upsilon(1 \mathrm{~S})$ state using a model with collisional damping, screening of the $b \bar{b}$ binding potential, gluon-induced dissociation, and hydrodynamic expansion of the fireball [2]. Concerning the $\Upsilon(2 S)$ state, however, the model underestimates suppression in peripheral collisions which indicates the need for further suppression mechanisms to be included. Promising candidates are magnetic field effects which are expected to possess the required centrality dependence.

Following the calculation of the electromagnetic fields generated in heavy ion collisions, we will make a first attempt to investigate their effect on the $b \bar{b}$ mesons produced in the collision by studying the magnetically induced mixing of the spin singlet and triplet states.

\section{Electromagnetic fields in heavy ion collisions}

We start by examining a single point charge $e$ moving with constant velocity $\boldsymbol{v}$ through a conducting quark-gluon medium. For a resting observer at position $\boldsymbol{r}=x \hat{\boldsymbol{e}}_{x}+y \hat{\boldsymbol{e}}_{y}+z \hat{\boldsymbol{e}}_{z}$ and time $t$, the charge's worldline is given by

$$
\boldsymbol{L}(t)=t \hat{\boldsymbol{e}}_{t}+v t \hat{\boldsymbol{e}}_{z}
$$

\footnotetext{
ae-mail: hoelck@thphys.uni-heidelberg.de
} 
where $\boldsymbol{v}=\boldsymbol{v} \hat{\boldsymbol{e}}_{z}$ and $\boldsymbol{L}(0)=\mathbf{0}$ by our choice of coordinates. For this observer, Maxwell's equations for the charge's electromagnetic fields $\boldsymbol{B}_{1}, \boldsymbol{E}_{1}, \boldsymbol{D}_{1}, \boldsymbol{H}_{1}$ take the form

$$
\begin{aligned}
\boldsymbol{\nabla} \cdot \boldsymbol{B}_{1} & =0 \\
\boldsymbol{\nabla} \times \boldsymbol{E}_{1} & =-\frac{\partial \boldsymbol{B}_{1}}{\partial t} \\
\boldsymbol{\nabla} \cdot \boldsymbol{D}_{1} & =e \delta(x) \delta(y) \delta(z-v t), \\
\boldsymbol{\nabla} \times \boldsymbol{H}_{1} & =\frac{\partial \boldsymbol{D}_{1}}{\partial t}+\sigma \boldsymbol{E}_{1}+e v \delta(x) \delta(y) \delta(z-v t) \hat{\boldsymbol{e}}_{z} .
\end{aligned}
$$

These equations can easily be solved in Fourier space but a re-transform to real space is infeasible since the dispersion relations of the quark-gluon medium's permittivity $\varepsilon$, permeability $\mu$, and conductivity $\sigma$ are unknown to date. To circumvent this problem, we adopt a very simple model for the medium's electromagnetic properties [3]: We neglect the medium's permittivity and permeability, i. e. set $\varepsilon \equiv \mu \equiv 1$, and characterise it by its finite conductivity only. The conductivity, in turn, is set to the constant value $\sigma=5.8 \mathrm{MeV}[3,4]$.

Using this simple model, exploiting cylindrical symmetry, and considering only high-relativistic settings $\left(\gamma_{v}=1 / \sqrt{1-v^{2}} \gg 1\right)$, we arrive at the approximate solution

$$
\begin{aligned}
E_{1}^{\rho} & =\frac{e}{4 \pi} \frac{\rho \sigma}{2 t_{\mathrm{eff}}^{2}} \mathrm{e}^{-\frac{\rho^{2} \sigma}{4 \mathrm{efff}^{\prime}}}, & B_{1}^{\rho} & =0, \\
E_{1}^{\varphi} & =0, & B_{1}^{\varphi} & =\operatorname{sgn}(v) E_{\rho}, \\
E_{1}^{z} & =\operatorname{sgn}(v) \frac{e}{4 \pi} \frac{\rho^{2} \sigma / 4-t_{\mathrm{eff}}}{\gamma_{v}^{2} t_{\mathrm{eff}}^{3}} \mathrm{e}^{-\frac{\rho^{2} \sigma}{4 \mathrm{e}_{\mathrm{eff}}}}, & B_{1}^{z} & =0, \\
\boldsymbol{D}_{1} & \equiv \boldsymbol{E}_{1}, & \boldsymbol{H}_{1} & \equiv \boldsymbol{B}_{1},
\end{aligned}
$$

where we use the shorthand $t_{\mathrm{eff}}=t-z / v$ and cylindrical coordinates $\rho=\sqrt{x^{2}+y^{2}}, \varphi=\operatorname{atan}(y / x)$.

One can think of a heavy ion nucleus as a nearly continuous distribution $n_{Z}$ of point charges $e$ normalised to the number of protons $Z$. At LHC beam rapidities, nuclei are Lorentz-contracted to almost 2-dimensional slabs. Thus, we model $n_{Z}$ as being localised in $z$-direction while choosing a Woods-Saxon shape in the $(x, y)$-plane,

$$
n_{Z}(\boldsymbol{r}) \propto \frac{\delta(z)}{1+\exp \left(\frac{\rho-R}{a}\right)},
$$

where $R$ and $a$ denote the radius and diffuseness of the nucleus, respectively. Due to the linearity of Maxwell's equations, the nucleus' electromagnetic fields $\boldsymbol{E}_{Z}, \boldsymbol{B}_{Z}$ are given by the convolution of $n_{Z}$ with the point charge fields $\boldsymbol{E}_{1}, \boldsymbol{B}_{1}$,

$$
\left[\begin{array}{l}
\boldsymbol{E}_{Z} \\
\boldsymbol{B}_{Z}
\end{array}\right](t, \boldsymbol{r})=\int \mathrm{d}^{3} r^{\prime} n\left(\boldsymbol{r}-\boldsymbol{r}^{\prime}\right)\left[\begin{array}{l}
\boldsymbol{E}_{1} \\
\boldsymbol{B}_{1}
\end{array}\right]\left(t, \boldsymbol{r}^{\prime}\right) .
$$

Since $n_{Z}$ is radially symmetric, $\boldsymbol{E}_{Z}, \boldsymbol{B}_{Z}$ and $\boldsymbol{E}_{1}, \boldsymbol{B}_{1}$ share the same cylindrical symmetries and vanishing components. As can be seen in Fig. 1, the general shape of the field components as functions of $\rho$ and $t_{\text {eff }}$ is not substantially altered by the convolution. The kink which can be seen in the plots of $\left|E_{1}^{z}\right|$ and $\left|E_{Z}^{z}\right|$ is the result of a change in sign occurring in the longitudinal field components when the point charge (or nucleus) passes by the observer. Due to time dilation, the observer perceives the sign 


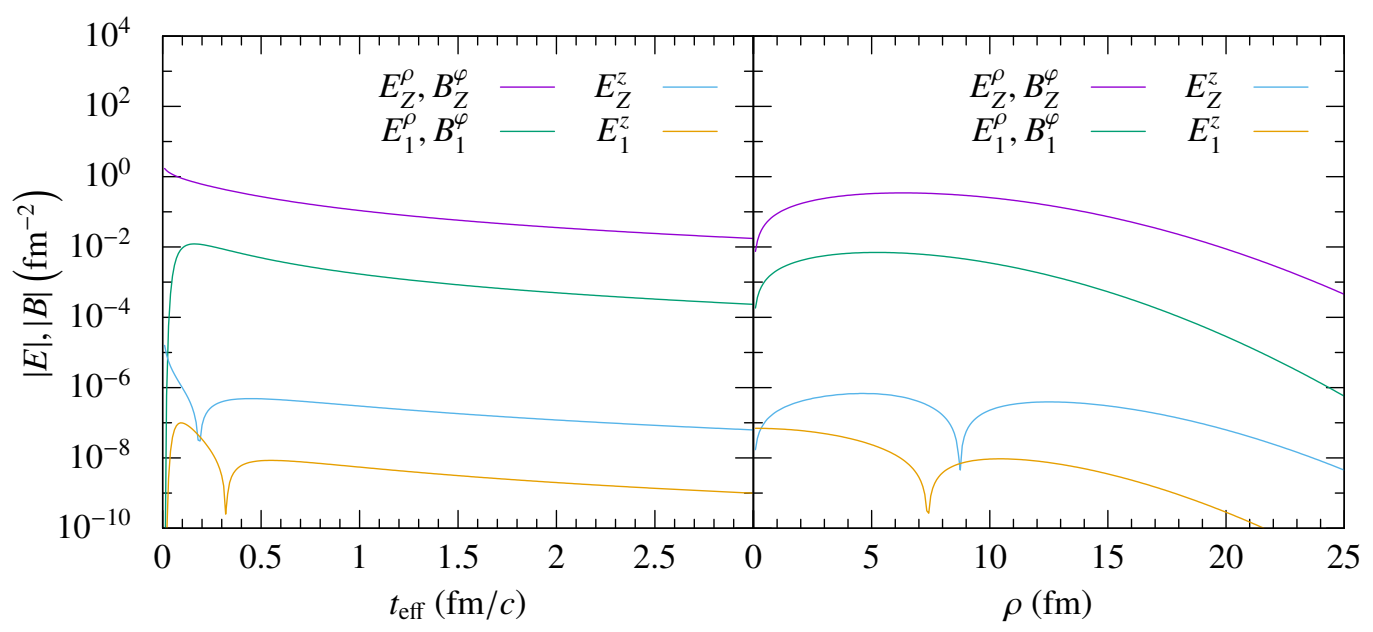

Figure 1. Electromagnetic field components for a single point charge and a $\mathrm{Pb}$ nucleus with $\operatorname{artanh}(v)=7.987$ as a function of $t_{\text {eff }}$ with $\rho=6.62 \mathrm{fm}$ (left) and as a function of $\rho$ with $t_{\text {eff }}=0.4 \mathrm{fm} / c$ (right).

change at $t_{\mathrm{eff}}>0$ for $\rho>0$. The exact point in time (and space) is slightly shifted for the convoluted fields because of the now finite-sized charge distribution.

By summing the fields of two antiparallel moving nuclei with velocities $\pm v_{\text {beam }}$, shifted in $x$-direction by the impact parameter $\boldsymbol{b}=b \hat{\boldsymbol{e}}_{x}$, we finally obtain the total electromagnetic field in a heavy ion collision,

$$
\left[\begin{array}{l}
\boldsymbol{E} \\
\mathcal{B}
\end{array}\right](t, \boldsymbol{r})=\left[\begin{array}{l}
\boldsymbol{E}_{Z} \\
\boldsymbol{B}_{Z}
\end{array}\right]_{v=+v_{\text {beam }}}(t, \boldsymbol{r}+\boldsymbol{b} / 2)+\left[\begin{array}{l}
\boldsymbol{E}_{Z} \\
\boldsymbol{B}_{Z}
\end{array}\right]_{v=-v_{\text {beam }}}(t, \boldsymbol{r}-\boldsymbol{b} / 2) .
$$

\section{Effective average field strength for $b \bar{b}$ mesons}

The electromagnetic field representations derived so far are only valid for observers resting in the coordinate system $(t, x, y, z)$ which can be identified with the laboratory frame in heavy ion collisions. To examine field effects on moving observers, we need to change to their rest frames and transform the fields accordingly.

If we neglect any transverse momentum, all $b \bar{b}$ mesons produced in the initial collision share the following properties:

- A constant velocity $\boldsymbol{u}$,

- movement parallel to the heavy ion nuclei, $\boldsymbol{u}=u \hat{\boldsymbol{e}}_{z}$, and

- a starting position in the $(z=0)$-plane at $t=0$, the time of the initial collision.

The restframe of this kind of observers coincides with the longitudinally co-moving frame (LCF) [5], which is reached through the coordinate transform $(t, x, y, z) \mapsto(\tau, x, y, \vartheta)$ with

$$
\tau=\sqrt{t^{2}-z^{2}}, \quad \vartheta=\operatorname{artanh}(z / t) .
$$

For the observers, the coordinate $\tau$ is always equal to their proper time $\sqrt{1-u^{2}} t=t / \gamma_{u}$ and the coordinate $\vartheta$ corresponds to their rapidity $\operatorname{artanh}(u)$. 


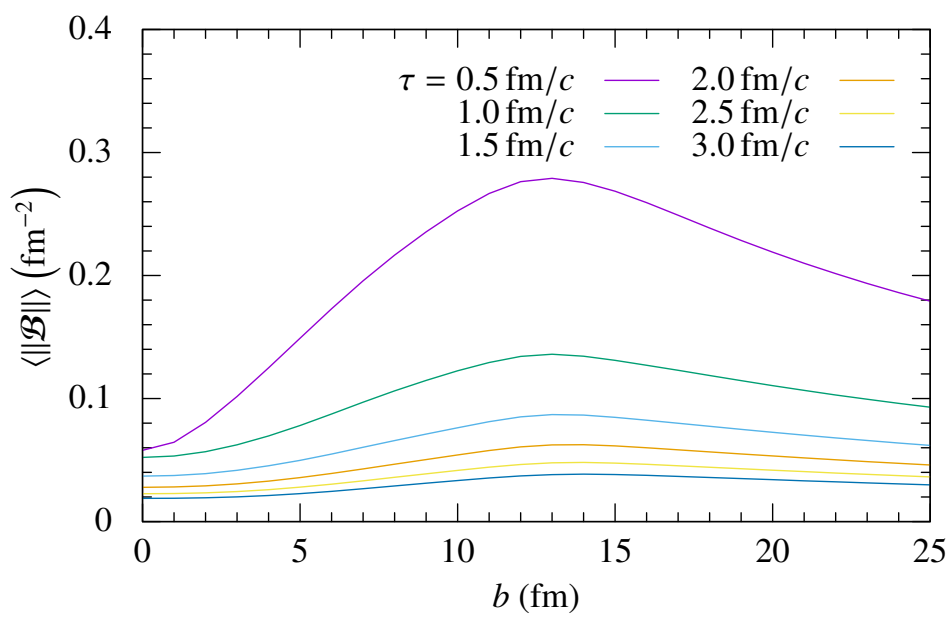

Figure 2. Average magnetic field strength $\langle\|\mathcal{B}\|\rangle$ in a $\mathrm{PbPb}$ collision with $\operatorname{artanh}\left(v_{\text {beam }}\right)=7.987$ (corresponds to $\left.\sqrt{s_{\mathrm{NN}}}=2.76 \mathrm{TeV}\right)$ as a function of the impact parameter $b$ at different proper times $\tau$ in the shared b $\bar{b}$ restframe. Converting natural to SI units, $1 \mathrm{fm}^{-2}$ corresponds to approximately $2 \times 10^{14}$ Tesla.

An electromagnetic field $\boldsymbol{E}_{\text {lab }}, \boldsymbol{B}_{\text {lab }}$ in the laboratory frame translates into the new coordinates as

$$
\begin{array}{ll}
E_{\mathrm{LCF}}^{x, y}=\left[\cosh (\vartheta) \boldsymbol{E}_{\mathrm{lab}}+\sinh (\vartheta) \hat{\boldsymbol{e}}_{z} \times \boldsymbol{B}_{\mathrm{lab}}\right] \cdot \hat{\boldsymbol{e}}_{x, y}, & E_{\mathrm{LCF}}^{\vartheta}=E_{\mathrm{lab}}^{z}, \\
B_{\mathrm{LCF}}^{x, y}=\left[\cosh (\vartheta) \boldsymbol{B}_{\mathrm{lab}}-\sinh (\vartheta) \hat{\boldsymbol{e}}_{z} \times \boldsymbol{E}_{\mathrm{lab}}\right] \cdot \hat{\boldsymbol{e}}_{x, y}, & B_{\mathrm{LCF}}^{\vartheta}=B_{\mathrm{lab}}^{z} .
\end{array}
$$

From our moving observers' perspective, this is equivalent to a Lorentz boost, since

$$
\cosh [\operatorname{artanh}(u)]=\gamma_{u}, \quad \sinh [\operatorname{artanh}(u)]=u \gamma_{u} .
$$

In the following, we will need an estimate of the magnetic field strength experienced by $b \bar{b}$ mesons produced in heavy ion collisions. For this purpose, we average the magnetic field magnitude with respect to the $\mathrm{b} \overline{\mathrm{b}}$ meson distribution $n_{\mathrm{b} \overline{\mathrm{b}}}$,

$$
\langle\|\mathcal{B}\|\rangle(\tau, b)=\frac{\int \mathrm{d} x \mathrm{~d} y \mathrm{~d} \vartheta n_{\mathrm{b} \overline{\mathrm{b}}}(x, y, \vartheta, b)\left\|\mathcal{B}_{\mathrm{LCF}}(\tau, x, y, \vartheta)\right\|}{\int \mathrm{d} x \mathrm{~d} y \mathrm{~d} \vartheta n_{\mathrm{b} \overline{\mathrm{b}}}(x, y, \vartheta, b)},
$$

where we have transformed $\mathcal{B}$ from Eq. (12) according to (14). We assume $n_{\mathrm{b} \overline{\mathrm{b}}}$ to be proportional to the number of nucleon-nucleon collisions $T_{A A}$ in real space and Gaussian-shaped in rapidity-space,

$$
n_{\mathrm{b} \overline{\mathrm{b}}}(x, y, \vartheta, b) \propto T_{A A}(x, y, b) \exp \left(-\frac{\vartheta^{2}}{2 \sigma_{\vartheta}^{2}}\right),
$$

where $\sigma_{\vartheta} \approx 2.7$ based on a fit of recent CMS data on LHC b $\bar{b}$ production [6].

In Fig. 2, the result is shown as function of the impact parameter $b$ and selected values of $\tau$. The portrayed time frame $0.5 \mathrm{fm} / c \leq \tau \leq 3.0 \mathrm{fm} / c$ is approximately equal to the lifetime of the quark-gluon medium, starting from the moment of $\mathrm{b} \overline{\mathrm{b}}$ meson formation. For fixed $\tau$, the magnetic field strength reaches its maximum at $b \approx 2 R$ when the two nuclei barely touch. For smaller impact parameters, the individual magnetic fields interfere destructively, whereas for large $b$ the nuclei field strengths decrease due to the large distances (cf. Fig. 1). With increasing proper time, $\langle\|\mathcal{B}\|\rangle$ decreases monotonically. 


\section{Magnetically induced $\Upsilon / \eta_{b}$ mixing}

Since bottom quarks are spin-1/2 particles, b $\bar{b}$ mesons exist in singlet $\left(\eta_{\mathrm{b}}\right)$ and triplet $(\Upsilon)$ spin configurations. ${ }^{1}$ We denote the latter by $\Upsilon^{0}, \Upsilon^{+}, \Upsilon^{-}$corresponding to the associated spin projection quantum number $m_{S} \in\{0, \pm 1\}$. In analogy to positronium, we assume a $b \bar{b}$ interacts with magnetic fields primarily by its quark spins coupling to the field [7] with a combined magnetic moment

$$
\hat{\boldsymbol{\mu}}_{\mathrm{b} \overline{\mathrm{b}}}=\frac{Q_{\mathrm{b}}}{m_{\mathrm{b}}} \hat{\boldsymbol{s}}_{\mathrm{b}}+\frac{Q_{\overline{\mathrm{b}}}}{m_{\overline{\mathrm{b}}}} \hat{\boldsymbol{s}}_{\overline{\mathrm{b}}}=\frac{Q_{\mathrm{b}}}{m_{\mathrm{b}}}\left(\hat{\boldsymbol{s}}_{\mathrm{b}}-\hat{\boldsymbol{s}}_{\overline{\mathrm{b}}}\right)
$$

subject to charge $Q_{\mathrm{b}}=-Q_{\overline{\mathrm{b}}}$, mass $m_{\mathrm{b}}=m_{\overline{\mathrm{b}}}$, and spin $\boldsymbol{s}_{\mathrm{b}}, \boldsymbol{s}_{\overline{\mathrm{b}}}$ of quark and antiquark. Hence, the Hamiltonian for a $b \bar{b}$ meson resting in a magnetic field $\boldsymbol{B}$ is given by

$$
\hat{H}=\left.\hat{H}\right|_{\boldsymbol{B}=\mathbf{0}}-\hat{\boldsymbol{\mu}}_{\mathrm{b} \overline{\mathrm{b}}} \cdot \boldsymbol{B}
$$

The eigenstates of $\left.\hat{H}\right|_{\boldsymbol{B}=\mathbf{0}}$ in spin space are well-known from its positronium analogue,

$$
\left|\eta_{\mathrm{b}}\right\rangle=\frac{|\uparrow \downarrow\rangle-|\downarrow \uparrow\rangle}{\sqrt{2}}, \quad\left|\Upsilon^{0}\right\rangle=\frac{|\uparrow \downarrow\rangle+|\downarrow \uparrow\rangle}{\sqrt{2}}, \quad\left|\Upsilon^{+}\right\rangle=|\uparrow \uparrow\rangle, \quad\left|\Upsilon^{-}\right\rangle=|\downarrow \downarrow\rangle .
$$

The symbols $|\uparrow \downarrow\rangle,|\downarrow \uparrow\rangle,|\uparrow \uparrow\rangle,|\downarrow \downarrow\rangle$ denote the spin alignments of the constituent quarks with the first (second) entry representing the (anti-)bottom quark spin. In the absence of magnetic fields, the spin part of $\hat{H}$ is isotropic and the actual orientation of $\uparrow, \downarrow$ irrelevant. If a field is present, we choose $\uparrow(\downarrow)$ to be equal (antiparallel) to the orientation of $\boldsymbol{B}$.

Treating $\hat{\boldsymbol{\mu}}_{\mathrm{b} \bar{b}} \cdot \boldsymbol{B}$ as a perturbation, we can compute the spin eigenstates of $\hat{H}$ for finite $\boldsymbol{B}$ [8],

$$
\left[\begin{array}{c}
\left|\Psi_{\mathrm{s}}\right\rangle \\
\left|\Psi_{\mathrm{t}}^{0}\right\rangle \\
\left|\Psi_{\mathrm{t}}^{+}\right\rangle \\
\left|\Psi_{\mathrm{t}}^{-}\right\rangle
\end{array}\right]=\left[\begin{array}{cccc}
\cos (\alpha) & -\sin (\alpha) & 0 & 0 \\
\sin (\alpha) & \cos (\alpha) & 0 & 0 \\
0 & 0 & 1 & 0 \\
0 & 0 & 0 & 1
\end{array}\right] \cdot\left[\begin{array}{c}
\left|\eta_{\mathrm{b}}\right\rangle \\
\left|\Upsilon^{0}\right\rangle \\
\left|\Upsilon^{+}\right\rangle \\
\left|\Upsilon^{-}\right\rangle
\end{array}\right]
$$

As can be seen, the perturbed and unperturbed eigenstate basis are related by a rotation matrix mixing the singlet and $m_{S}=0$ triplet states. The mixing angle $\alpha$ depends on the energy difference and magnetic momentum of $\eta_{\mathrm{b}}$ and $\Upsilon^{0}$,

$$
\tan (\alpha)=\frac{x}{1+\sqrt{1+x^{2}}}, \quad x=\frac{2 \boldsymbol{\mu} \cdot \boldsymbol{B}}{\left|E_{\eta_{\mathrm{b}}}-E_{\Upsilon^{0}}\right|}, \quad \boldsymbol{\mu}=\left\langle\eta_{\mathrm{b}}\left|\hat{\boldsymbol{\mu}}_{\mathrm{b} \overline{\mathrm{b}}}\right| \eta_{\mathrm{b}}\right\rangle=\left\langle\Upsilon^{0}\left|\hat{\boldsymbol{\mu}}_{\mathrm{b} \overline{\mathrm{b}}}\right| \Upsilon^{0}\right\rangle
$$

The $m_{S}= \pm 1$ spin states remain unaffected, $\left|\Psi_{\mathrm{t}}^{+}\right\rangle=\left|\Upsilon^{+}\right\rangle,\left|\Psi_{\mathrm{t}}^{-}\right\rangle=\left|\Upsilon^{-}\right\rangle$, since

$$
\left\langle\Upsilon^{+}\left|\hat{\boldsymbol{\mu}}_{\mathrm{b} \overline{\mathrm{b}}}\right| \Upsilon^{+}\right\rangle=\mathbf{0}=\left\langle\Upsilon^{-}\left|\hat{\boldsymbol{\mu}}_{\mathrm{b} \overline{\mathrm{b}}}\right| \Upsilon^{-}\right\rangle
$$

To get an idea of the order of magnitude of the mixing, we roughly approximate

$$
\boldsymbol{\mu} \cdot \boldsymbol{B} \approx\|\boldsymbol{\mu}\|\langle\|\mathcal{B}\|\rangle
$$

using our estimate of the average magnetic field strength derived in Eq. (17). In Fig. 3, the overlap

\footnotetext{
${ }^{1}$ Strictly speaking, the names $\eta_{\mathrm{b}}$ and $\Upsilon$ only refer to states with angular momentum $L=0$, while states with $L=1$ are termed $\mathrm{h}_{\mathrm{b}}$ (singlet) and $\chi_{\mathrm{b}}$ (triplet), respectively. However, in the following, we will use the labels $\eta_{\mathrm{b}}$ and $\Upsilon$ for all states regardless of $L$ to keep notation simple.
} 


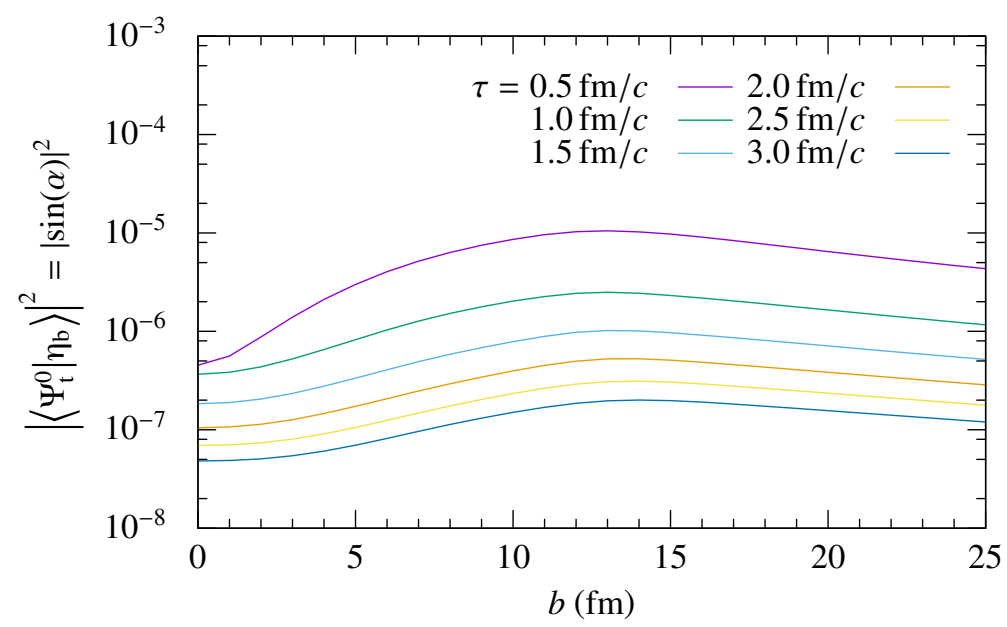

Figure 3. Overlap between the spin states $\left|\Psi_{\mathrm{t}}^{0}\right\rangle$ and $\left|\eta_{\mathrm{b}}\right\rangle$ with $N=1, L=0$ as function of the impact parameter $b$ at different proper times $\tau$ using $\langle\|\mathcal{B}\|\rangle$ as magnetic field strength.

of the $\Psi_{\mathrm{t}}^{0}$ and $\eta_{\mathrm{b}}$ spin states $\left|\left\langle\Psi_{\mathrm{t}}^{0} \mid \eta_{\mathrm{b}}\right\rangle\right|^{2}=|\sin (\alpha)|^{2}$ is plotted as a function of the impact parameter $b$ and proper time $\tau$. Being a monotonous function of $\langle\|\mathcal{B}\|\rangle$, the general shape of the overlap is similar to Fig. 2. However, even for the $(b, \tau)$ configuration yielding the highest magnetic field strength, the overlap is $\lesssim 10^{-5}$ and therefore negligible. In consequence, the magnetically perturbed states $\Psi_{\mathrm{s}}, \Psi_{\mathrm{t}}^{0}$ in relativistic heavy ion collisions are expected to behave virtually identically to their unperturbed counterparts $\eta_{\mathrm{b}}, \Upsilon^{0}$.

\section{Outlook}

Due to exposure to strong magnetic fields, the $b \bar{b}$ mesons in relativistic heavy ion collisions might behave differently from the ones produced in pp events where almost no electromagnetic fields are present. The magnetically perturbed spin singlet state $\left|\Psi_{\mathrm{s}}\right\rangle$ and $m_{S}=0$ triplet state $\left|\Psi_{\mathrm{t}}^{0}\right\rangle$ are each composed of a superposition of the two unperturbed spin states $\left|\eta_{\mathrm{b}}\right\rangle$ and $\left|\Upsilon^{0}\right\rangle$. Hence, properties of the perturbed states related to spin, such as the decay widths between excited states and the final decay into dimuon pairs, can differ compared to their unperturbed counterparts. However, the extent of this effect is found to be negligible at LHC energies.

Therefore, future investigation will focus on other possible interactions between $\Upsilon$ mesons and electromagnetic fields. Especially the fields' impact on $b \bar{b}$ formation and thereby the initial meson populations could prove important since the highest field strengths occur immediately after the collision where meson formation takes place. In addition, photon-gluon induced dissociation of $b \bar{b}$ pairs and dissociation due to increased potential energy may significantly affect in-medium decay widths for the excited $b \bar{b}$ states.

\section{References}

[1] S. Chatrchyan, et al., Phys. Rev. Lett. 109, 222301 (2012)

[2] F. Nendzig, and G. Wolschin, J. Phys. G Nucl. Part. Phys. 41, 095003 (2014) 
[3] K. Tuchin, Phys. Rev. C 88, 024911 (2013)

[4] H.-T. Ding, A. Francis, O. Kaczmarek, F. Karsch, E. Laermann, and W. Soeldner, Phys. Rev. D 83, 034504 (2011)

[5] J. D. Bjorken, Phys. Rev. D 27, 140 (1983)

[6] M. Jo, et al., Proc. Quark Matter Conf. Kobe 2015, in preparation; CMS-PAS-HIN-15-001

[7] P. Filip, Proc. Sci. CPOD 2013, 035 (2013)

[8] A. Rich, Rev. Mod. Phys. 53, 127 (1981) 